\section{Adrenal incidentaloma: when should I worry?}

\section{Carmel T. Mooney}

In human medicine, adrenal incidentalomas are defined as masses found serendipitously at diagnostic imaging without prior evidence of overt disease. Increasingly, in dogs, adrenal incidentalomas are found during either abdominal ultrasonography or computed tomography (CT) when other non-endocrine diseases are being investigated. Overall the prevalence is between $4 \%$ and $9 \%$, depending on imaging modality (ultrasonography or CT) and they are typically found in older animals. They are usually unilateral, although bilateral masses have been described.

These masses may represent metastatic disease or primary adrenal tumours that can be benign or malignant and functional or non-functional. Functional tumours may produce cortisol, cortisol precursors, aldosterone or catecholamines.

Once identified, a systematic approach should be taken for diagnosis and prognosis. Metastatic adrenal gland disease is not uncommon, occurring in over $20 \%$ of dogs with various malignancies, affecting one or both adrenal glands with approximately equal frequency. Typically when adrenal glands are affected, disseminated disease is present. Thus adrenal incidentalomas that represent metastatic disease are commonly seen in animals being examined for neoplasia.

Deciding on whether primary adrenal masses are benign or malignant can be difficult. Evidence of local spread and vascular invasion may help but is uncommon. The larger the mass $(>2 \mathrm{~cm})$ the greater the likelihood of malignancy. Bilateral adrenal enlargement with an additional mass like lesion could represent pituitary dependent asymmetric adrenal hyperplasia, although concurrent pituitary dependent hyperadrenocorticism and an adrenal tumour cannot be ruled out. Atrophy of the contralateral gland (maximum width $<0.5 \mathrm{~cm}$ ) suggests cortical hyperfunction arising from the affected gland.

Further investigations should be directed at assessing the functional nature of the masses. Careful evaluation of the history, clinical examination and routine clinicopathological analyses is important as some clinical features may have gone unnoticed or misinterpreted. Diagnostic testing should be based on any pertinent findings. In the absence of significant abnormalities, blood pressure measurement is important as it may indicate the presence of hyperadrenocorticism, hyperaldosteronism or phaeochromocytomas.

Systematic evaluation of cases without obvious clinical abnormalities includes assessment for hyperadrenocorticism and phaeochromocytoma. In the latter case, the low dose dexamethasone suppression test is preferred because of its high negative predictive value. However, the adrenocorticotropic hormone (ACTH) response test may provide useful information indicating a possible cortisol precursor secreting tumour. Plasma free normetanephrine and the urinary normetanephrine to creatinine ratio are helpful for diagnosing phaeochromocytoma.
Hyperaldosteronism is rare in dogs and usually accompanied by overt clinical signs. Measurement of aldosterone in association with plasma renin activity is required for diagnosis.

In many cases, despite intensive investigations, there is limited evidence of hyperfunction or malignancy. Although benign lesions are likely, malignancy cannot be ruled out. These cases should be reassessed at regular intervals, starting 4-6 weeks after initial presentation and every 3-6 months thereafter depending on progression. For those cases that are likely to have malignant disease or that have functional lesions, surgical removal is recommended.

\section{KEY LEARNING OBJECTIVES}

- Be able to prioritize differentials for adrenal incidentalomas

- Be confident in selecting and interpreting appropriate diagnostic tests for investigation

- Increase confidence in discussing prognosis with owners

\section{MULTIPLE CHOICE QUESTIONS}

1. Following abdominal ultrasonography in a dog a right-sided adrenal mass of $4 \mathrm{~cm}$ is identified with the maximal width of the left adrenal gland at $0.4 \mathrm{~cm}$. What is the next best diagnostic test?
(A) Low-dose dexamethasone suppression test
(B) High-dose dexamethasone suppression test
(C) Urine cortisol:creatinine ratio
(D) Dexamethasone suppression of urine cortisol:creatinine ratio

2. A mildly hypertensive dog has a right-sided adrenal mass of $1.8 \mathrm{~cm}$ and normal left-sided adrenal gland measurements. Cortisol concentrations before and 3 and 8 hours following low-dose dexamethasone are $250 \mathrm{nmol} / \mathrm{l},<27.6 \mathrm{nmol} / \mathrm{l}$ (reference interval $<27.6 \mathrm{nmol} / \mathrm{l}$ ) and $75 \mathrm{nmol} / \mathrm{l}$ (reference interval $<27.6 \mathrm{nmol} / \mathrm{l})$. Plasma free normetanephrine and urine normetanephrine:creatinine concentrations are $25 \mathrm{nmol} / \mathrm{l}$ (reference interval $<10 \mathrm{nmol} / \mathrm{l}$ ) and 1458 (reference interval <400). What is the most likely diagnosis?
(A) Hyperadrenocorticism
(B) Phaeochromocytoma
(C) Either hyperadrenocorticism or phaeochromocytoma
(D) Both hyperadrenocorticism and phaeochromocytoma

3. A hypertensive cat has a $2 \mathrm{~cm}$ left-sided adrenal mass. What is the next best test to perform to achieve a diagnosis?
(A) Plasma normetanephrine concentration
(B) ACTH response test
(C) Circulating aldosterone concentration
(D) Aldosterone to renin ratio 